\title{
A closer look at Deep-fibre FTTdp
}

\author{
Craig Watkins \\ Informative Technology Innovations \\ Kelvin Lillingstone-Hall \\ OAK Telecom
}

\begin{abstract}
Summary: In this paper we outline a number of matters that have been raised in relation to Deep-fibre Fibre-to-the-Distribution-Point (FTTdp), and address practical ways that FTTdp can be expected to deliver a maximum overall cost-benefit outcome for the Australian NBN. We present brief discussions of some HFC (Hybrid Fibre Coax) and FTTN (Fibre-to-the-Node) issues considered of concern, to place FTTdp in perspective. We touch briefly on the threat from wireless communications to the fixed line technologies. We discuss the FTTdp equipment ecosystem, migration from legacy services to FTTN or FTTdp, in-building copper concerns, and LFN architecture savings emerging from the FTTdp approach. As previously, we conclude that FTTdp must be honestly evaluated if the nation is to achieve a maximal NBN capability outcome.
\end{abstract}

\section{Introduction}

The vision of the NBN is to supply access network capacity to economically meet demands of today and those of the future. Deep-fibre Fibre-to-the-Distribution-Point (FTTdp) in the form of fibre to the street lead-in pit at the front fence, with an average copper lead-in of $30 \mathrm{~m}$, promises a level of overall network capability very close to that of full FTTP (Fibre to the Premises). Improvements in fibre-related technologies continue to promise increasing levels of connectivity (Ovum 2014). Simultaneously, while improvements in fibre technologies and construction practices continue to reduce fibre deployment costs, deployment of customer lead-in connections is more resistant to the potential for cost savings. Following our earlier paper (Watkins and Lillingstone-Hall 2014), we believe that deep-fibre FTTdp is a strong candidate for delivering the maximum cost-benefit access network outcome to the nation.

Communications technology determinations are highly contingent upon access network bandwidth demand projections, and these are observed to vary immensely. A sensible analysis exploiting valid assumptions and rational logic can eliminate the most offensive demand projection outliers, but there is no obvious way to obtain significant consensus perspective on future demand requirements of the NBN. We can, however, expect to obtain strong consensus on the fact that demand variability from user to user is high. We can also 
be quite certain that bandwidth demands will continue to rise for the foreseeable future. Translation of these facts to NBN deployment determinations implies the need for high network flexibility.

Fibre to the Node (FTTN) is a well-known path to incremental improvements in access network capability. With augmentation by a small number of micro-node devices, FTTN is capable of delivering significant connectivity improvements over exchange-based ADSL services. The best improvement can be expected for those users closest to nodes or micronodes. It is, however, problematic to provide any certainty in connectivity outcomes for premises more distant from node locations. Copper-related factors are an important consideration for all non-fibre connection solutions, and in-building or in-premises copper configuration issues may cause a need for expensive rectification work. The long-term prospects for FTTN are also poor in the sense that demand growth may quickly outstrip the capability of long copper pairs from the node, with the only prospect being eventual deployment of fibre beyond the node.

Advanced technologies such as G.fast (ITU 2013) offer little benefit in a FTTN setting (although vectored G.fast may prove useful with the related FTTB (Fibre-to-theBuilding/Basement) approach in some MDU environments). The recently announced Vplus technology from Alcatel-Lucent (Russell et al 2014) appears to maximise the capability of DSL-based technologies in a node setting. It is important to note that even with Vplus, only premises close to the node receive significant benefit. The geometry of Telstra Distribution Areas may limit meaningful Vplus potential gains from the node to less than $30 \%$ of premises. Vplus allows a distribution area to be subdivided by strategic positioning of smaller node devices to reduce overall copper loop lengths, but the deployment expense of such an option must be compared with the relative merits of a deep-fibre FTTdp solution.

Deep-fibre FTTdp, bringing fibre to street distribution pits, eliminates around $90 \%$ of the copper network compared to FTTN, and importantly eliminates practically all copper network joints. The considerably shorter copper lengths of deep-fibre FTTdp maximise the prospects for technologies such as G.fast, although extremely good connectivity outcomes are also readily achieved through VDSL2, where 200 Mbps download and 50 Mbps upload are likely to be achievable for a vast majority of premises. We note that VDSL2 offers an aggregate (download plus upload) of 300 Mbps for short copper lengths (Lantiq 2014, Hirscher 2014).

FTTdp provides three significant advantages in overcoming in-building or in-premises copper configuration issues compared to FTTN:

(1) a large reduction in overall copper length maximises connection throughput capabilities even with undesirable copper configuration conditions (bridge taps); 
(2) significant reduction in bundled copper usage reduces the prospect that undesirable signals such as impulse noises collected in one premises will impact connectivity in other premises; and

(3) highly problematic premises are provided with an economic option to upgrade to a fibre drop connection, effectively capping in-premises copper rectification expenses.

The core advantages of the deep-fibre FTTdp approach are that it facilitates user demand flexibility and accommodates large potential future bandwidth demand growth. The deepfibre FTTdp approach provides a base-level of connectivity to all users that is substantially higher than the average connectivity level provided via FTTN. This has significant value in relation to the capacity of the network to meet future demand scenarios. It is crucial to note that lower demand growth projections may not value such capability appropriately, and the precise terms of any Statement of Expectations given to NBN Co becomes a critical issue for the future of the nation's communications infrastructure.

FTTdp economically provides for the high connectivity demands of a small proportion of key consumers. It does this in a way that does not stifle creativity and growth at the start-up enterprise level where there may be limited ability to pay for more expensive connectivity outcomes. Limits on the ability to pay may come from cash-flow restrictions, or the expectation of future growth-demanded business premises relocation.

Deep-fibre FTTdp provides an overall level of network capability similar to that of FTTP, yet with cost and time equations comparable with FTTN, due to elimination of the extreme expense and labour resource requirements associated with FTTP premises connections in the Australian NBN context. It promises long-term communications infrastructure for the nation, at a substantial cost and time reduction to FTTP, making a superior national communications outcome possible.

This paper extends the presentation of deep-fibre FTTdp introduced by the authors in a March 2014 paper published in the same journal. We cover a collection of concerns and issues that have been raised through the ensuing six months discussing the rationality of FTTdp within wide circles. Our primary resolve that FTTdp must be considered seriously as a mainstream option for NBN deployment is significantly stronger today than in March. We believe a highly capable NBN outcome is achievable for the nation within appropriate cost and time constraints through deep-fibre FTTdp.

\section{A definition of deep-fibre FTTdp}

There is no single definition of a copper network "distribution point". As a consequence, FTTdp can have a variety of meanings. FTTdp is associated with larger "micro"-node devices of from 16 to 48 ports, as adopted in some deployment scenarios overseas. We are primarily 
interested in the deep-fibre FTTdp concept, where fibre is deployed to the street lead-in pit. Small micro-node devices of 4 or 8 ports supply premises over existing copper lead-in twisted pairs from the pits. The average copper length is thus perhaps as little as $30 \mathrm{~m}$.

Deep-fibre FTTdp in this sense can be directly compared to the NBN Co FTTP architecture, with multi-port units being replaced by small reverse-powered (powered from premises over the lead-in copper) 4 or 8 port weather-protected (including immersion protection) micronode devices mounted inside the lead-in pit. The associated reduction in fibre counts (a single fibre connects each micro-node device to the Fibre Distribution Hub instead of a multiple-fibre ribbon in the NBN Co FTTP architecture) provides flexibilities that minimise the need for pit and pipe rectification work. The avoidance of fibre premises lead-in deployment (by FTTdp exploitation of existing twisted pair copper lead-ins) allows substantial deployment cost and time savings. From the NBN Co Strategic Review (NBN Co 2013), a brownfields per-premises cost of $\$ 3260$ can be derived for the "Radically Redesigned FTTP” scenario. The per-premises fibre connection cost of $\$ 2100$ is largely avoided by FTTdp. (Connection costs remain, related to micro-node devices, customer premises equipment, and pit installation of FTTdp micro-node equipment, although these likely total a small percentage only of the $\$ 2100$ savings.)

The deep-fibre FTTdp approach results in a significant reduction in fibre count at the FDH (Fibre Distribution Hub). Ultimately this allows for a FDH enclosure that is greatly reduced in size, minimising street impact, and potentially allowing for pit-retractable designs.

For practical reasons related to efficient deployment, single port VDSL or G.fast devices may also be part of the deep-fibre FTTdp solution set. It should be clear that the lack of shared lead-ins from many pits implies that there is little need for vectoring implementation because there will be negligible cross-talk present. Consequently, serving multiple customers from a pit via single port devices is feasible, as is the adoption of 4 or 8 port micro-node equipment that may not implement vectoring functionality (where significant crosstalk and high line utilisation factors exist, vectoring becomes instrumental in delivering a superior overall outcome).

The deep-fibre FTTdp approach is a Passive Optical Network architecture up to the premises lead-in. As such, it is trivially upgradable on an individual premises basis to a FTTP connection. An NBN construction program based on FTTdp remains a "single build" solution to supplying the long-term communications demands of the nation. The cost and time savings in comparison to FTTP are extremely positive, and may translate favourably to a direct positive comparison between FTTdp and FTTN. VDSL2-based FTTdp, at circa 250 Mbps down and 50 Mbps up (or similar) provides a much higher level of baseline 
connectivity than FTTN, and crucially provides economic means of connectivity upgrade for those premises requiring more.

This deep-fibre FTTdp approach, taking fibre to street lead-in pits, potentially aligns with the naturally emergent "fibre edge" of the network. An all-fibre network (FTTP) represents a particular vision of the future that many suggest is an inescapable reality on a long-term basis. However, there is no guarantee that the economics of fibre premises connections will compete with DSL approaches for connectivity demand levels that we can be confident of seeing from the vast majority of premises. We can, however, be certain that the fibre edge of the network will be significantly beyond the node (Telstra Distribution Area). Fibre running down local streets may thus be the natural edge of the fibre network, although another five years of progress might be necessary to provide any conclusiveness to such a prediction.

Deep-fibre FTTdp provides robust and flexible connectivity capabilities, economically meeting demands of all users, serving long-term network requirements, and avoiding the considerable cost of tailored civil works to deploy new fibre lead-ins to every premises. Heritage buildings are one example where concerns about the physical infrastructure associated with fibre lead-in deployment is a very 'sensitive' topic. In a more general view, the need for involved civil works through individual premises is a deployment situation that quickly leads to high cost and time delay. Existing, suitably short copper lead-ins, enable high connectivity outcomes with relatively minimal expense via VDSL2 or other related DSL technologies.

\section{Deep-fibre FTTdp compared to HFC}

The NBN Co Strategic Review (NBN Co 2013) strongly suggested that the economics of exploiting existing Hybrid-Fibre-Coax (HFC) networks was sound. Almost 12 months later, little information has emerged from NBN Co to support the assertions of very low incremental costs to supply NBN services over extant HFC infrastructure. However, we must assume that there is merit to careful consideration of how the existing HFC assets might best be exploited.

John Goddard (Goddard 2014) outlines many of the elemental concerns related to incorporation of HFC assets in the NBN product mix. However, a concrete engineering roadmap for HFC assets is yet to emerge from NBN Co. Large node sizes are a primary challenge for existing HFC assets to supply high connectivity levels across a wide customer base in the NBN application. The node size issue is seen as particularly relevant in conjunction with the likely addition of many new premises connecting to the HFC network within existing physical node boundaries. (As affordable and capable NBN services are provided over the HFC networks it is sensible to imagine a significant proportion of ADSL users will switch to 
cable-modem NBN service in these areas.) The overlap of two distinct HFC network architectures is likely to present additional challenges.

More pragmatic matters are expected to relate to the difficulties in simultaneous operation of multiple networks over the HFC infrastructure (for example Foxtel and NBN) and the related issue of which entity retains update control of relevant CPE (Customer Premises Equipment). The installed capability of the HFC backbone fibre network, and the ready extensibility of the downstream fibre infrastructure (ease of node splitting and driving fibre deeper into the network) are key details that only a few select individuals are likely to properly appreciate. The difficulty is not that a path forward does not exist. CableLabs standards and global cable industry best practice provide the necessary capability augmentation route. A lack of complete appreciation of the current state of the HFC networks, and the associated uncertainty of upgrade cost estimates is the key concern. Ultimately the difficulty and costs associated with NBN Co exploitation of the HFC infrastructure may be dramatically in excess of those suggested by the 2013 NBN Strategic Review.

As HFC infrastructure was initially deployed, the goal of supplying as many as 2000 premises on a node would have been seen as an impressive engineering achievement. We don't claim that the average number of premises per HFC node is as high as this, and note that Goddard suggests 200 is a more sensible figure. A percentage of HFC nodes may of course contain significantly more than the average number of premises. A high number of premises connected to a node is a present and future engineering challenge for data service delivery. GPON architectures typically settle on maximum sharing of the access network across 32 premises (the NBN Co approach doesn't even fully populate to this level initially). This small number of premises per node represents an efficient engineering trade-off between anticipated demand growth, expected active component upgrade cycles, and the cost of deployment of even higher fibre counts throughout the LNDN (Local Network/Distribution Network). While HFC networks may never require node sizes as small as this, a massive reduction in node size is likely to be necessary on a relatively short timeframe. High levels of sharing have a very real impact on overall Quality of Service. The quantum of work required to deliver sensible node sizes to HFC networks must ideally be contrasted with the alternatives involving new LFN (Local Fibre Network) deployments (one such option being the deep fibre FTTdp approach).

Allowing that use of HFC assets may have a valuable role to play in the NBN, HFC coverage infill areas might also present prospects for FTTdp. We should note that aerial cabling deployment options would most likely favour HFC infrastructure or FTTP. NBN Co CEO Bill Morrow, when discussing HFC recently (Dobbie 2014), appears to suggest that the cost of aerial customer connection is so low that we must rightfully ask whether aerial FTTP must AUSTRALIAN JOURNAL OF TELECOMMUNICATIONS AND THE DIGITAL ECONOMY VOLUME 2 NUMBER 4, DECEMBER 2014 ISSN 2203-1693. Copyright (C) 2014 The Author(s). http://doi.org/10.7790/ajtde.v2n4.67 
once again be more widely considered for the NBN. However, Bill Morrow's comments might also ultimately be found to be overly optimistic in this regard.

Even a $20 \%$ proportion of premises lacking a lead-in may translate to favourable economics for alternative approaches in HFC coverage areas. Clearly detailed costing analysis is required prior to making any solid conclusions in this regard. However, we note that history of the Australian cable TV and cable modem market sectors, combined with competing ADSL offerings, implies that many premises lead-ins would need to be constructed in the NBN HFC scenario. As lead-ins are added there is risk of DOCSIS (Data Over Cable Service Interface Specification) node overloading, contributing to declining service levels without additional HFC network investment. We must be realistic in admitting that while some premises report good cable modem service today, there are many reports of unsatisfactory service, especially in relation to peak usage time periods.

This paper focuses on deep-fibre FTTdp in contrast to FTTN. For similar investments of cost and time, FTTdp provides significant capability increase over FTTN. Importantly, FTTdp avoids the relative lack of an upgrade path from FTTN to higher capability outcomes. Any comparison between HFC and FTTdp is expected to be less conclusive than a comparison between FTTN and FTTdp. Some of the primary flexibility advantages of FTTdp compared to FTTN are loosely paralleled by abilities to incrementally upgrade HFC outcomes. This does not imply that detailed analysis won't determine FTTdp to be an economic option in HFC areas, but that our focus at present is a comparison between FTTN and deep-fibre FTTdp. We assume that, a priori, exploitation of $\mathrm{HFC}$ assets by the NBN is economically rational, but note that determinations in this regard must weigh long-term considerations in addition to more immediate concerns, and consider network flexibility in meeting the needs of highdemand customers. Without input to the contrary, FTTdp is perhaps best considered as a baseline alternative option in HFC areas.

\section{The upgrade path from FTTN}

FTTN is reported as being capable of delivering 100 Mbps (downstream, and perhaps 40 Mbps upstream) at copper distances of up to 300 to 400 metres from the node. For larger distances the data rate decreases, but it is suggested that 50 Mbps may be possible at a distance of up to 80om from the node (VanHastel \& Verlinden 2012). Of course, the reality of what is achievable depends on both the specific condition of the copper network from the node to the premises, and any in-building or in-premises copper configuration issues. We discuss the latter concern in more detail in a subsequent section.

The recent Alcatel-Lucent Vplus announcement (Russell et al 2014) promises improvement in capability over the first few hundred metres of copper loop length through exploitation of 
a $30 \mathrm{MHz}$ VDSL2 bandwidth from the node. However, as mentioned in the introduction above, obtaining advantage from Vplus, for more than a moderate percentage of premises within a node area, is likely to require deployment of smaller satellite nodes. The marginal cost versus benefit analysis for deployment of such nodes appears relatively unappealing compared to other options such as deep-fibre FTTdp, although the option clearly deserves closer analysis if only as a deployment reference case. How quickly Vplus can be adopted by the broader VDSL modem manufacturer ecosystem also remains to be seen. Even a requirement for a future flash upgrade of CPE will severely limit the ease of future FTTN augmentation via Vplus deployment.

For the $17 \mathrm{MHz}$ VDSL2 deployment likely to be provided as part of the NBN, a minimum 50 Mbps download connection, coupled with an appropriate upload allowance of perhaps $20 \mathrm{Mbps}$, (note NBN Co is still focussed on a lower minimum of $25 \mathrm{Mbps}$ download) offers a dramatic improvement in capability compared to average ADSL experience of around $6 \mathrm{Mbps}$. Most users would be happy with $50 \mathrm{Mbps}$ (down) today, and there is good reason (Department of Communications 2014a) to suggest that there is limited overall willingness to pay for more (using analyses rooted in our current experience).

The NBN must, however, look to the future. The minimum horizon of interest is the several years to deploy the NBN, but a 10 to 30 year outlook is also necessary for such a national infrastructure project. While it is obvious that projections of bandwidth demand growth vary tremendously, there is only a need to look forward a few short years before we start to appreciate that the risk of being locked in to a $50 \mathrm{Mbps}$ connectivity outcome is substantial. We can be certain, globally, that $4 \mathrm{~K}$ video streaming to multiple concurrent devices per premises will become a reality for a significant number of premises within a very small number of years. While available CPU and memory capabilities allow some continuation of advances in video compression technology, we must conservatively assume a $4 \mathrm{~K}$ video data rate of between 20 and 30 Mbps. The NBN is not simply a video entertainment system; however it would be an extreme mistake to underestimate the risk of locking the nation into a 50 Mbps outcome. We must ask what economic pathways exist to provide significant improvements in connectivity, assuming the nation has already deployed FTTN.

Within the FTTN model, there is a real prospect of exploitation of pair bonding to provide higher connectivity outcomes for a small proportion of premises that require improved bandwidth. This option might be expected to be of first interest to premises most distant from the node. There is a risk of spare pairs being exhausted quickly, even allowing for a reasonable percentage of premises disconnecting from the wired network (some Telstra Distribution Areas will fare better in regard to spare pairs than others). It is also not clear whether extant FTTN equipment is fully capable of seamless pair bonding on a significant scale. Assuming node equipment supports bonding and NBN Co software systems are up to AUSTRALIAN JOURNAL OF TELECOMMUNICATIONS AND THE DIGITAL ECONOMY VOLUME 2 NUMBER 4, DECEMBER 2014 ISSN 2203-1693. Copyright (C) 2014 The Author(s). http://doi.org/10.7790/ajtde.v2n4.67 
the task, we must also ask whether the additional copper management and maintenance requirements make this option economic. Any need to re-patch pairs at the node to ensure they are served by the same line card for bonding purposes (if we assume this might be a requirement), would rapidly detract from the economics of provisioning incremental bandwidth improvements from the node by pair bonding. Note that vectoring is readily able to occur across line cards, facilitated by exchange of a modest amount of vectoring data. It is difficult, but not impossible, to imagine a related data exchange process that would facilitate bonding of pairs served by different line cards.

There is also a prospect within the FTTN approach to gradually migrate to deeper fibre deployment by installation of micro-nodes of from 16 to 48 ports (as suggested by, but not limited to, the Vplus concept mentioned above), directly replacing ports serviced from the node. However, we might quickly imagine that such an approach has limited practical appeal. To reach any worthwhile number of such mid-size 'nodes', enough fibres would be radiating from the node to pass the street pits of a significant number of premises within the Distribution Area. The alternative approach of a GPON-based deep-fibre FTTdp solution has quite obvious appeal where this is the case, delivering a markedly improved overall network connectivity result.

The inescapable conclusion is that any significant upgrade to connection outcomes beyond what is possible today with vectored VDSL2 from the node, must necessarily involve a substantial deployment of fibre past the node. The prime candidate to achieve this is the deep-fibre FTTdp model where the twisted-pair copper lead-in network is exploited for the final few metres from the street pit to the premises.

FTTN provides a very poor economic outlook for any fibre-on-demand product offering from NBN Co. Demand variability, coupled with the low (or modest) level of capability supplied by FTTN, suggests latent demand for fibre-on-demand will be significant. However, the cost of provisioning such a service is likely to suppress such demand, potentially to the point of extinguishing it (the economics of fibre-on-demand within the FTTN model look spectacularly poor once limitations of the total pool of skilled staff are considered along with the need for NBN Co to undertake a special project for the benefit of just a few customers).

The failure to deliver a practical fibre-on-demand solution translates to a significant reduction in the time frame to when it becomes necessary to drive fibre past the node. The substantial sunk investments at the node provide no benefit to driving fibre past the node other than the fact that CPE at the customers' premises should provide an almost seamless transition to VDSL-based FTTdp. The only additional CPE requirement should be the provision of reverse power feed from premises to the micro-node devices in the lead-in street pit. Expensive FTTN cabinets with sophisticated cooling systems, mains powering, battery 
back-up, and time-consuming copper patching work to existing node pillars, have an extremely short potential service life based on most (if not all) sensible demand-growth projections. At risk of repetition, while uncertainty about copper condition exists in determining what level of capability is provided by FTTN, the Shannon-Hartley theorem provides clear guidance on the maximum level of connectivity able to be achieved. We are unable to be as certain on the topic of future demand projection, but reasonable growth projections suggest there is little safety margin associated with FTTN delivery capability.

The considerable cost of provision and installation of FTTN cabinets, including the deployment time factor due to the high-level of technician skill required on the copper network, would translate to an appreciable amount of fibre deployment past the node in a lean FTTdp LFN (Local Fibre Network) model. Furthermore, exhausting the nation's available equity funding to deploy fibre only to the node level, risks the prospect of additional funding being absent should an upgrade from FTTN be required to meet growing demand in the near future. Clearly we are not able to determine what real appetite for equity funding the nation possesses, and the $\$ 29.5$ billion indicated in the April 2014 NBN Co Statement of Expectations (Department of Communications 2014b) does not necessarily reflect the nation's equity limit. The key point is the rationality of careful determination on expenditure that provides a limited future benefit. Such expenditure may be justifiable, but without systematic due diligence the nation can not necessarily claim it is justified expenditure.

\section{The migration challenge}

Migration of users and services from the existing copper network to any NBN network presents a number of challenges that must be considered carefully from an overall systems engineering perspective. The issue of how to optimally allow cut-over for a large number of independent end-users, operating via a broad collection of RSPs (Retail Service Providers), and making NBN connection decisions at varying times, is a major concern. It is not difficult to imagine that options relating to systems flexibility in regard to migration approaches, are likely to translate to enormous overall systems deployment cost differentials. A primary consideration is the cost of a truck roll and minimising the total number of necessary truck rolls within practical constraints of workable (and available) support software (OSS Operations Support Systems, and BSS - Business Support Systems).

It is important that a holistic systems engineering perspective is taken with regard to the cost of truck rolls. The use of NBN Co marketing power to negotiate favourable contracts may translate to an NBN Co book cost of truck rolls that is below a sustainable level to the independent contractors working at the installer level. Pushing systemic inefficiencies of truck movements between widely varying locations onto the independent contractors may cause a false valuation of key engineering options that might otherwise provide an overall 
benefit to the NBN. Implemented cost structures that prove unsustainable are ultimately likely to be identified and rectified. The most substantial risk is where costing errors are instrumental in deployment decisions, and the crucial point is that a holistic engineering perspective should aim to avoid such errors. The complexity of the migration challenge ensures this remains a non-trivial task.

Legacy services operating over the copper network also present significant migration challenges. The NBN Co approach to solving both this and the basic migration problem has centred on allowing an 18 month window where the new NBN FTTP network is operated in parallel with the existing copper infrastructure. A similar parallel network scenario is possible with both FTTN and FTTdp. In both cases copper connectivity is readily provided to the exchange for legacy telephony services including ISDN by the use of small line filter/splitter devices. Maintaining legacy ADSL services presents somewhat more challenge.

The VDSL standard has been designed to be interoperable with ADSL, with effectively identical modulation format over the ADSL band. Consequently VDSL implementations readily provide ADSL connectivity. However, migration of individual RSP ADSL services supplied from the exchange to the node or micro-node would most certainly be a non-trivial undertaking. Fortunately the impact of the ADSL bandwidth on VDSL vectoring is comparably minor, allowing legacy ADSL services to be supplied from the exchange as vectored VDSL is provided from the node. A disadvantage of this approach is that new VDSL service connections require attendance at the node for copper connection. Fortunately it is possible to pre-deliver a customer VDSL modem that will default to the customer's ADSL configuration until such time that the new VDSL connection is established, minimising the service disruption experienced by the end user. Of course this requires access to legacy ADSL username and password settings that most end users do not have at their fingertips, but presumably competitive RSPs will seize the opportunity to deliver superior customer service.

Further improvements in the migration approach that reduce the overall level of truck rolls may be possible through judicious use of splitter devices that allow ADSL services to be served from the exchange initially. Even non-ideal or partial solutions to the migration challenge that allow greater batching of work at the nodes, are likely to make a major contribution to the NBN bottom line. We note that G.fast is designed to interoperate with legacy ADSL and VDSL services through optional notching out the lower portion of the transmission bandwidth as required. The greater modulation bandwidth of VDSL2, at $17 \mathrm{MHz}$ for FTTN and $30 \mathrm{MHz}$ for FTTdp, compared to the $2 \mathrm{MHz}$ bandwidth of ADSL, enables similar operation, even if the original VDSL standard appears to have not anticipated such a deployment scenario (the authors have not been able to identify specific VDSL2 bandwidth profiles designed to support this use). 


\section{LFN architecture for FTTdp}

Consideration of FTTdp quickly reveals prospects for major LFN architecture change compared to that already considered by NBN Co for FTTP (NBN Co 2012). Deep-fibre FTTdp is most obviously closely aligned with a multi-stage split LFN architecture. Compared to the base case of the NBN Co FTTP architecture, a significant reduction in fibre counts is obtained.

NBN Co Type 2.1 architecture runs 12-fibre ribbon cables from the FDH (Fibre Distribution Hub) in what is effectively a star configuration. The "radically redesigned FTTP" scenario introduced in the NBN Co Strategic Review reduces the overall fibre count, and we can easily speculate that this architecture might entail use of 6-fibre ribbon cables, but we ignore this as the base comparison case due to lack of specific published detail.

Cables of size varying from 72 to 288 fibres are used in the Type 2.1 architecture for the LFN. The 72-fibre cable has six ribbons of 12 fibres each, and is thus capable of supplying six multiport devices, presumably in six different pits. The 288-fibre cable likewise serves up to 24 street pits. Directly translating this to the FTTdp situation, we would presume the need for LFN cables of between 6 and 24 fibres. Such smaller and lighter fibres present a greatly improved outlook for ease of physical deployment. There is no primary difference in the optical budget or physical reach of the GPON (Gigabit Passive Optical Network) system in the two LFN architectures of FTTP or FTTdp.

Without an individual fibre from each premises back to a centralised FDH cabinet, there is a minor loss in capability with regard to balancing the load across individual GPON circuits. In the scenario where FTTdp micro-node devices remain the dominant connectivity medium for a significant duration, the concern of loss in such 'perfect' flexibility is moot. With a centralised primary stage split architecture we still retain the ability to balance GPON circuits to the level of the micro-node device. In reality the benefit arising from balancing GPON circuits beyond this level is only likely to be applicable to very high usage customers. Such customers would presumably be prepared to pay modest additional fees to have priority access to select reduced fan-out or otherwise low use GPON circuits, facilitated by spare fibre capacity in the LFN cables. We also need to appreciate the fact that the level of future need for GPON circuit balancing may be offset by other fibre-optic technologies such as the use of additional wavelengths over the existing PON (Ovum 2014).

A small amount of additional fibre capacity in LFN cables enables an extremely low-cost option where balancing GPON circuits above the level of the micro-node devices becomes desired. Such might be envisaged where neighbouring high-demand small business enterprises share FTTdp service from the same pit. Noting that the FTTdp GPON link is able 
to support an average of $100 / 40 \mathrm{Mbps}$ service for 25 simultaneous users, it is only where an individual premises upgrades to a fibre (or G.fast link) and utilises this at high capacity for a large fraction of time that statistical GPON limitations are likely to be experienced. Two such high-demand premises served from neighbouring pits presents no difficulty in the centralised splitter model for balancing the GPON circuits. When such high demand users are served from a single pit, a spare fibre can be used to allow the premises to be served on separate GPON circuits.

Perhaps more radically, a non-centralised primary splitter architecture is able to reduce LFN fibre counts even further. In the more extreme case, a primary 8-way split, with micro-nodes serving on average 4 premises, 6 fibres can maximally serve 24 pits. Being this lean on the fibre count does potentially impact our ability to easily balance GPON circuits, and such a fully distributed primary split architecture perhaps has little overall value. On the other hand, some element of non-centralised primary splitter architecture may be of interest, and we attempt to elucidate this in the following paragraphs.

A fully connectorised splitter array in a central location under the FTTdp model is reduced in size by at least a factor of 4 compared to the NBN Co FDH approach (splitter unit size is effectively determined by number of connectors). Design attention focussed on minimal physical size is likely to provide further benefit, and the prospect of a primary splitter array that is fully retractable into a pit becomes entirely feasible. The elimination of street furniture impact and the avoidance of damage from errant vehicles, etc., is a positive result that merits serious consideration. However, a splitter architecture fully mounted in existing pit infrastructure (thus providing substantial deployment cost and time savings) becomes possible once a very small distribution of splitter components is allowed.

The physical fan-out of fibre bundles from a FDH location provides for locations where a small number of splitter units might be readily mounted in a semi-distributed fashion. Excess fibres linking the distributed splitter locations can supply the ability to balance GPON loads to the limited extent that this might be deemed an important capability. While it may seem anathema to those heavily indoctrinated in the NBN architecture as seen to date, the use of fully spliced splitters is also worth consideration. Technologies such as spider-web ribbon (Takeda 2014) make it possible to tease out a single fibre in the situation where it becomes desirable to migrate users from one splitter to another. A departure from Nielsen's Law exponential demand growth (Nielsen 1998), to more conservative bandwidth demand growth projections, suggests that the need for shuffling users between splitters might be rare enough that the overheads of a fully connectorised solution are entirely unjustified.

The advantages of having a single small, flexible fibre cable option for deployment in the LFN are obvious. A 24-fibre spider-web ribbon solution (in say six groups of 4 fibres) 
provides ample convenience and flexibility to meet the demands of any particular LFN layout. The supply logistics side of the deployment is greatly simplified by such an approach. Some fibre network nodes in the LFN may naturally suggest multiple fibre cables deviating at the node, while others may be optimally suited to insertion of splitters. Whether ultimately using field-installable connectors, fusion-spliced connectors, or direct-splice connections, tapping into a single fibre cable variant is a skill quickly mastered by the NBN Co installer army. A clear division of labour is possible between teams laying fibre, those connecting passive network devices, and those connecting the active micro-node devices.

For low take-up areas there is unlikely to be any advantage to pre-installation of micro-node devices in pits prior to service orders being received. In the case of a single-port FTTdp device being deployed, there is obviously no reason to pre-install the active equipment. (The use of single port active components presumably requires a second-stage splitter installed at the pit with at least the second service delivered from the pit, or requires additional LFN fibre capacity and a reversion to the larger centralised split approach of the existing NBN Co FDH architecture.)

In summary, there are many reasons to expect significant LFN fibre deployment gains to be achieved with the deep-fibre FTTdp paradigm. The gains are importantly both in terms of cost and deployment time, compounding the cost and time gains made by avoiding fibre premises drops.

\section{The FTTdp equipment ecosystem}

FTTN equipment is of sufficient overall complexity to provide a largely prohibitive barrier to market entry for many otherwise capable suppliers. While no single element of a node device provides inordinate difficulty for a new market entrant, to be able to provide sales of volume to offset engineering development costs, a high level of customer confidence must be created in all elements. The established market players thus have a considerable advantage in FTTN node equipment.

An associated issue is the reduced prospect of a mixed vendor model for FTTN node equipment. Different vendors are likely to provide units that are functionally equivalent at a primary level, but from an installation and operational perspective, any collection of subtle differences translates to a potentially large cost overhead in dealing with multiple equipment configurations. Ultimately this reduces the number of suppliers actively exploited by NBN Co, and has an impact on the ability of NBN Co to negotiate favourable equipment pricing from any major vendor. However such issues are not without precedent in telecommunications networks, and ways to (at least partially) mitigate such effects are possible. 
In contrast, the small micro-node devices of deep-fibre FTTdp present a far-simplified set of possible specifications. The prospects for exploiting functionally identical equipment from a large range of suppliers is very real. The overall simplicity of micro-node devices ensure that the production of a superior product is within reach of many companies, and there is relatively little advantage gained by the more traditional equipment suppliers in this space. Examples to illustrate the level of activity in this sector of the industry are the young start-up Sckipio (Sckipio 2014), and the partnership announced at the October 2014 Broadband World Forum between TE Connectivity and Technicolor (Technicolor 2014). Ultimately the intense activity in this sector of the market translates to likely availability of equipment at reasonable prices and high functionality, today or in the very near future, with a steady improvement in the supply equation expected over the coming years.

The passive-cooling requirement for small micro-node devices ensures that minimisation of power consumption is a primary design requirement. Lantiq (Lantiq 2014) indicates a single-port device may consume 6 Watts. A reasonable expectation is that multi-port devices would have an improved consumption figure on a per-user basis, but even at 6 Watts it is clear that the "green credentials" of the deep-fibre FTTdp option are positive.

We must note that NBN Co might benefit from leading market specifications for FTTdp micro-node product solutions. There is little risk from doing so for such a comparatively simple component, and the upside benefit could be significant. Alternatively, the global industry is likely to supply highly capable devices that closely match the needs of the Australian NBN, even though application of deep-fibre FTTdp may differ slightly in overseas deployment settings.

The high cost of labour in the Australian context produces an inordinately high cost of fibre connections to premises. In turn this suggests that the prospects for deep-fibre FTTdp are very positive in the NBN setting. A reduced deployment cost of fibre premises drops in overseas settings may suggest that the economics of FTTdp are less positive. However, in environments where duplicate or competing local connectivity infrastructure exists, competitive forces from the end-user perspective, or the reduced possibility of sufficiently long-term end-user commitments, are likely to also drive deployment of deep-fibre FTTdp options. Such overseas deployments provide a healthy FTTdp industry ecosystem, and is thus good news for the Australian NBN.

The recent October 2014 Broadband World Forum in Amsterdam, revealed significant FTTdp announcements from Alcatel-Lucent (Alcatel-Lucent 2014), TE Connectivity/ Technicolor (Technicolor 2014), and Sckipio (Sckipio 2014). Other well-known suppliers such as Huawei (Techweek 2013) and Adtran (Adtran 2014) are also active in the FTTdp 
product space. It is clear that there are positive global movements towards deep-fibre FTTdp, with strong reasons to expect rapid developments in this market over the next two years.

\section{The in-building copper configuration issue}

In-Building or in-premises copper configuration can be a significant issue for DSL-based technologies. The prospect of bridge tap configurations is high in some building and premises settings. The detrimental impact bridge taps have on the overall spectral response of the DSL channel can be enormous. In-building copper wiring can also pick up large amounts of impulse noise, with this being repetitive impulse events in many cases. Crosstalk in shared copper bundles can translate impacts from impulse noise from one premises to other premises in a small percentage of cases.

Many modern premises operate with a single Telstra socket outlet employed for DSL modem/router and cordless phone. The ideal DSL outcome would be achieved in the situation where the Telstra socket employed for such purpose was the first Telstra entry point in the premises, with the remainder of the in-building copper wiring disconnected. Any alteration of in-building wiring work is a licensed cabling activity and thus requires professional attendance inside premises or to common building areas in the MDU (Multiple Dwelling Unit) setting. While the cost of such copper rectification work may be readily passed to premises owners or bodies corporate, it still represents a real cost component of the NBN. Additionally, any skilled staffing requirement needed for such network-edge deployment tasks is likely to extract suitable staff from the core NBN network deployment pool, ultimately raising costs and slowing the deployment.

It is important to appreciate that access to in-building wiring may be a simple task in some situations. Perhaps the most simplistic case is where the first Telstra socket (Telstra copper entry point) is opened to simply disconnect any additional household twisted pair wiring. Some percentage of cases can be expected to have wiring junctions more deeply buried within the building structure, whether that is within walls, above ceilings, or under floors. Tragedies related to the recent home insulation scheme should remind us that the cost of professional rectification works can rise sharply due to Occupational Health and Safety/ Work Health and Safety precautions in many situations.

In the MDU setting an additional difficulty is that cooperation may be required between premises owners and the body corporate. While it is likely that the desires of many premises owners would be similar, and hence the bodies corporate should be agreeable, there will be some cases where this ideal is not readily obtained. In the situation where access to all building risers is necessary to fix systemic building copper issues, the prospect of installing new building cabling of some variety (CAT6, Coax, or Fibre) needs consideration. It would be 
a massive undertaking for NBN Co to expect to deliver a single optimised service to all MDUs in this regard. However, the scope for specialised industry providers targeting such services in a competitive industry fashion, directed by bodies corporate, is exciting.

As FTTN and FTTdp are both reliant on DSL, it is natural to expect that they both involve similar costs associated with in-building copper rectification works. However, there are reasons to expect that the FTTdp scenario obtains a significant reduction in the overall costs of such rectification work. Firstly, the ability to economically route a new fibre service from the street pit effectively caps the in-building copper rectification cost. Secondly, the very short FTTdp copper length provides maximisation of the DSL connection capability even in the presence of in-building bridge taps or poor copper joints. Thirdly, the reduced use of shared copper bundles inherent in FTTdp translates to a marked reduction in the potential for adverse impacts on other premises to be generated by undesirable wiring configuration in any single premises (such as where significant impulse noise is collected).

Importantly, any reduction or delay in the call on licensed cabling professionals to perform in-building copper rectification works, allows such skilled staff to be employed in the core NBN build.

An additional prospect with FTTdp is the potential for a significant reduction in the need for licensed cabling activities, assuming appropriate changes to regulations. While a need for regulation and licensing of cable installers will continue with FTTdp, it is reasonable to expect that this may be at an appreciably reduced level. In-building cabling has a smaller association with the broader communications network under FTTdp, and the associated risk of detrimental impact is considerably reduced (albeit non-zero). Ultimately any efficiencybased simplification of cabling regulation and licensing can help to widen the overall pool of skilled resources, improving the cost and time outlook for the national NBN build. The weighting to any benefit that might be obtained in this area is low due to the fact that regulation changes are assumed to be necessary, and an optimal migration strategy may involve a long-term connection of the in-premises copper network to the existing exchange network, thus negating any potential for significant regulation and license simplification.

\section{The threat from wireless networks}

There is little disagreement that wireless and wire-based (predominantly fibre) networks provide complementary services. The promise of improved wireless connectivity continues to lure a selection of media commentators into thinking that wireless is a panacea. The reality is that many premises will retain a need for a fixed-network connection. A growing number of premises may be able to satisfy their demands (usually where these demands are 
significantly lower than average) solely and economically by wireless access provided by nonNBN Co commercial entities. This presents a threat to all NBN Co access technologies.

The large capital investment (and time investment) in FTTP implies that the technology evolution risk from wireless development is significant. Any reduction in revenue as users migrate away from the NBN is particularly problematic in the FTTP model where premises may connect (incurring the hefty connection costs for NBN Co) and yet within as little as two years determine that they are better served by commercial (non NBN Co) wireless services. The elevated ARPU (Average Revenue per User) required by NBN Co limits its ability to minimise such losses through competitive pricing.

The lower overall deployment cost and time for FTTN and FTTdp network options presents an improved picture. However, the proportion of premises abandoning the NBN in favour of commercial mobile wireless services can be expected to be higher in the situation where the NBN is seen to provide limited additional value. The quantum of risk is greatest in the FTTN case, where although monthly NBN data quotas are likely to be higher than commercial mobile services for the foreseeable future, peak download rate is likely to be a more meaningful determinant of service quality for many users.

The argument as to the relative importance of peak download rate versus streaming throughput capability is a very complex one, on which we are unlikely to be able to obtain significant consensus at present. The recently completed NBN Cost Benefit Analysis (Department of Communications 2014a) can certainly be excused for largely neglecting the consideration. The graphics shown on page 107 and 167 of the CBA report can be interpreted as accommodating the issue of peak download rate via translation by 'expert' opinion to an equivalent streaming rate. The $15 \mathrm{Mbps}$ shown for software updates illuminates the point. In many cases software update download occurs in the background and a download rate as low as a fraction of percent of the stated $15 \mathrm{Mbps}$ would suffice. However, there are other situations where a software update prevents work from commencing or continuing, and in this case update responsiveness is a crucial usability factor. For the purposes of the CBA Choice modelling, 15 Mbps appears to be sensible compromise figure, but it clearly risks oversimplifying the analysis.

Of more material impact on the outcomes of the CBA choice analysis is clearly the figure shown for $4 \mathrm{~K}$ TV streaming. This is shown on page 107 as $30 \mathrm{Mbps}$, yet is modified on page 167 (as used in the choice study) to $20 \mathrm{Mbps}$. The present point is that it is clearly a difficult task to obtain reliable input on streaming throughput requirements. It is probably prudent to remain open to the prospect that high peak data rates may provide benefits that are even more difficult to attempt to quantify. The threat from wireless networks is that by providing high peak data rates ( $5 \mathrm{G}$ Wireless, etc.), there may be a significant associated usability 
improvement experienced by some users. The $25 \mathrm{Mbps}$ minimum suggested in the NBN Co Statement of Expectations is low enough that it may cause concern in this way.

Optimal NBN Co deployment decisions must weigh the likely threat from wireless advances. It is suggested that a proper analysis will lean toward the higher capabilities of the deep-fibre FTTdp option as part of considering the future wireless threat.

\section{Conclusion}

For brownfield underground distribution areas, the deep-fibre FTTdp approach supplies overall network capability effectively identical to that provided by FTTP at a substantially reduced cost. In greenfield areas and in overhead distribution areas, reduced benefits (in the sense of limited cost improvements compared to FTTP) are provided by deep-fibre FTTdp, leading to the probable preference for full FTTP in these areas.

It is less clear that FTTdp has a significant role to play in HFC areas. However, while the basic promise of exploiting existing HFC infrastructure is sound, practical realities may ultimately dictate that FTTdp has a role, even if in niche areas within the overall HFC footprint. Certainly FTTdp, and FTTP in aerial deployment areas, should be considered as baseline cases against which any HFC investment is compared. HFC investment based solely on immediate returns may incur a risk of inferior long-term outcomes. Short term priorities must be weighed appropriately with an understanding of network flexibility and broader lifecycle costs and benefits.

Outside of HFC deployment areas at least, the deep-fibre FTTdp approach fosters a substantial re-think of the LFN architecture. The prospect for dramatic cost and time savings in this part of the deployment is enormous and exciting in terms of the overall NBN outcome. The LFN architecture ideas introduced within this paper are not intended to be suggestive of an optimal approach, as there are many factors to consider in determining an ideal architecture. However, the ideas proposed should help to delineate some of the many considerations that require more careful analysis by NBN Co or a related entity charged with maximising deployment outcomes. A field trial by NBN Co of FTTdp installations would also help to focus on the optimal approach to detailed deep-fibre FTTdp architecture.

The Deep-fibre FTTdp approach has validity well beyond that of the Australian context. However, some peculiarities of the Australian deployment environment (primarily high labour costs, relative lack of competing infrastructure, and large Telstra distribution areas) suggest that this form of FTTdp is likely to have particular applicability to our nation's NBN. By fully embracing FTTdp, the nation may achieve an optimised NBN outcome of which we can be justifiably proud. 


\section{References}

Adtran, 2014. 'Accelerating Gigabit Broadband: A FTTdp White Paper', Adtran, 2014, available at: https://www.adtran.com/web/fileDownload/doc/32246, retrieved October 24, 2014

Alcatel-Lucent, 2014. 'Alcatel-Lucent's new G.fast solution to help operators provide more subscribers with ultra-broadband access', Alcatel-Lucent Press Release, October 21, 2014, available at: http://www.alcatel-lucent.com/press/2014/alcatel-lucents-new-gfastsolution-help-operators-provide-more-subscribers-ultra-broadband-access, accessed October 24, 2014

Russell, K; Spruyt,P; VanHastel, S. 2014. 'Vplus gets more out of VDSL2 vectoring', AlcatelLucent TECHzine, October 16, 2014, available at: http://www2.alcatellucent.com/techzine/vplus-gets-vdsl2-vectoring, accessed November 22, 2014

Department of Communications. 2014a. 'Independent cost-benefit analysis of broadband and review of regulation: Volume II - the costs and benefits of high speed broadband', available at:

http://www.communications.gov.au/ data/assets/pdf file/0003/243039/CostBenefit Analysis - FINAL - For Publication.pdf, August 27, 2014, retrieved September 3, 2014.

Department of Communications. 2014b. 'Statement of Expectations', April 8, 2014, available at:

http://www.communications.gov.au/ data/assets/pdf file/o014/221162/SOE Shareh older Minister letter.pdf, retrieved April 14, 2014.

Dobbie, Phil. 2014. 'Bill Morrow with NBN Co's reality check', Crosstalk, October 23, 2014, available at: http://phildobbie.com/main/podcasts/crosstalk/item/1343-bill-morrowwith-nbn-s-reality-check, accessed October 24, 2014

Goddard, John. 2014. 'The Untapped Potential of Hybrid Fibre Coaxial Networks', Australian Journal of Telecommunications and the Digital Economy 2(2) pp. 17.1-17.7, DOI: 10.7790/ajtde.v2n2.40. Available at: http://telsoc.org.au/journal, accessed October 24, 2014

Hirscher, Stefan. 2014. '300 Mbps over Copper: VDSL2 FTTdp Delivers the Future, Today', available at:

http://www.lantiq.com/fileadmin/products/201404 Lantiq VDSL FTTdp with VINA $\underline{\mathrm{X}} \mathrm{TM}$ dp.pdf, retrieved October 24, 2014

ITU. 2013. 'New ITU broadband standard fast-tracks route to 1Gbit/s: G.fast capitalises on existing copper to deliver fibre speeds at lower costs', ITU Press Release, December 11, 2013, available at:

http://www.itu.int/net/pressoffice/press releases/2013/74.aspx\#.VHAUglWUfCI, accessed November 22, 2014

Lantiq. 2014. '300 Mbps VDSL Right Now - Lantiq Introduces New Fiber-to-theDistribution-Point Solution', available at: http://www.connectionsplus.ca/pressreleases/300-mbps-vdsl-right-now-lantiq-introduces-new-fiber-to-the-distributionpoint-solution/, accessed October 24, 2014

NBN Co. 2013. Strategic Review, Final Report (with redactions). Available at: http://www.nbnco.com.au/content/dam/nbnco/documents/NBN-Co-Strategic-ReviewReport.pdf, retrieved December 12, 2013

NBN Co. 2012. Network Design Rules. Available at: http://www.nbnco.com.au/content/dam/nbnco/documents/network-design-rules2012.pdf, retrieved November 22, 2014 
Nielsen, Jakob. 1998. 'Nielsen's Law of Internet Bandwidth', available at http://www.nngroup.com/articles/law-of-bandwidth/, accessed January 12, 2014

Ovum. 2014. 'TWDM PON Is on the Horizon: Facilitating fast FTTx network monetization' Ovum, 2014, available at:

http://info.ovum.com/uploads/files/TWDM PON white paper.pdf, retrieved October 24, 2014.

Sckipio. 2014. 'World's First 16-Port G.fast Demonstration Shown by Sckipio at Broadband World Forum', Sckipio Press Release, October 21, 2014, available at: http://www.sckipio.com/worlds-first-16-port-g-fast-demo, accessed November 24, 2014

Takeda D; Isaji, M; Tomikawa, K; Osato, K; Yamanaka, M; Okada, N. 2014. 'Ultra-HighDensity Optical Fiber Cable', Fujikura Technical Review, 2014, available at: http://www.fujikura.co.jp/eng/rd/gihou/backnumber/pages/ icsFiles/afieldfile/2014/ 07/04/43e 02.pdf, retrieved November 22, 2014

Technicolor. 2014. 'Technicolor: Frontrunner in next-gen ultra broadband technologies that boost the speed of your network'. Available at:

http://newsletterstack.technicolor.com/workspace/uploads/images/next-gen-ubbtechnologies-final.pdf, accessed November 22, 2014

TechWeek Europe. 2013. 'BT To Trial Huawei G.fast FTTdp Copper Broadband Technology'. available at: http://www.techweekeurope.co.uk/news/huawei-gfast-fttdp-copper-bt129855, accessed January 8, 2014

VanHastel, S; Verlinden, J. 2012. 'VDSL2 Vectoring Delivers on its Promise'. Alcatel-Lucent TECHzine, September 17, 2012, available at: http://www2.alcatellucent.com/techzine/vdsl2-vectoring-delivers-on-its-promise, accessed November 22, 2014

Watkins, C; Lillingstone-Hall, K. 2014. 'The FTTdp technology option for the Australian National Broadband Network', Australian Journal of Telecommunications and the Digital Economy 2(1), March 2014, available at: http://doi.org/10.7790/ajtde.v2n1.26 Available at: http://telsoc.org.au/journal, accessed October 24, 2014 